\title{
Efectos industriales de la política monetaria en México: un enfoque de vectores autoregresivos estructurales en panel ${ }^{1}$
}

Víctor Hugo Torres Preciado*

Reyna Vergara González**

Miguel ÁNgel Tinoco ZeRmeÑo***

\section{RESUMEN}

El objetivo de esta investigación es analizar el efecto que un cambio permanente en la política monetaria tendría en las actividades manufactureras en México. Se argumenta que debido a la importancia económica de este tipo de actividades en el crecimiento económico y la creación de empleo en el país, es necesario conocer el impacto de la política en este sector. A partir de un modelo vectorial estructural en panel propuesto por Pedroni (2013), los resultados indican que los choques no anticipados en la política monetaria tendrían un efecto diferencial sustantivo entre las diferentes actividades manufactureras, situación que tiene implicaciones acerca del canal a través del cual el banco central puede incidir en la estabilización de precios e inclusive en la economía real.

Palabras clave: política monetaria, industria manufacturera, panel VAR estructural, México.

Clasificación JEL: C32, E32, E52, L60.

Los autores agradecen los comentarios de los dictaminadores anónimos.

* Profesor-investigador de tiempo completo, Facultad de Economía, Universidad de Colima, México. Correo electrónico: tpreciado04@gmail.com.

** Profesora-investigadora de tiempo completo, Facultad de Economía, Universidad Autónoma del Estado de México, México. Correo electrónico: rvergarag@uaemex.mx.

*** Profesor-investigador de tiempo completo, Facultad de Economía, Universidad de Colima, México. Correo electrónico: mangel.tinoco@gmail.com. 


\section{ABSTRACT \\ INDUSTRIAL EFFECTS OF MONETARY POLICY IN MEXICO: A STRUCTURAL PANEL VECTOR AUTOGRESSIÓN APPROACH}

The aim of this investigation consists in analyzing the effect that a permanent change in the monetary policy may exert on the Mexican manufacturing activities. It is argued that given the economic importance still present in the manufacturing industry for economic growth and employment, it is necessary to know the impact of monetary policy on this sector. By implementing a structural panel VAR model based on Pedroni (2013)'s approach, the results indicate that unanticipated shocks of monetary policy would have substantive differential effects across the manufacturing activities, with implications regarding the channel by which the central bank would achieve price stabilization and influence the real economy.

Keywords: monetary policy, manufacturing industry, structural panel VAR, Mexico.

JEL Classification: C32, E32, E52, L60.

\section{INTRODUCCIÓN}

El estudio de las implicaciones económicas de la política monetaria ha sido objeto de interés permanente en el ámbito académico y práctico, particularmente en la evaluación de su efectividad para la consecución de los objetivos de inflación establecidos por la banca central, el análisis de los diferentes canales de transmisión y su efecto esperado en las variables económicas reales. Aunque estos aspectos han sido abordados en la literatura macroeconómica, diferentes estudios señalan la importancia de profundizar en el conocimiento de los efectos que la conducción de la política monetaria puede ejercer en el desempeño económico cuando se consideran diferentes niveles de desagregación geográfica e inclusive sectoriales.

En particular, la relevancia de ahondar en los efectos que las variaciones de la política monetaria ejercen en las economías regionales ha sido señalada inicialmente en el ámbito de las uniones monetarias, como en el caso de la Unión Europea, donde el reconocimiento de la 
heterogeneidad de las estructuras económicas de los países miembros ha sido parte de un largo debate en el diseño e instrumentación de una política monetaria común. No obstante, el interés por identificar los efectos geográficos derivados de esta política también ha sido recogido por países no miembros que consideran relevante conocer si existen efectos diferenciados a escala regional, para posteriormente determinar las fuentes potenciales de este comportamiento diferenciado en asociación con la estructura industrial predominante, argumentando que la política monetaria es potencialmente más efectiva cuando existe una mayor concentración de actividad manufacturera y de pequeñas empresas.

No obstante, aunque este argumento es generalmente aceptado, también impide observar si la actividad industrial responde asimétricamente a las variaciones en la política monetaria. Al respecto, algunos estudios se han enfocado en averiguar si la actividad económica industrial reacciona de forma diferenciada ante las variaciones de la política monetaria, aspecto fundamental para identificar desde el punto de vista sectorial cuáles actividades son más sensibles y, por tanto, a través de cuáles sectores la instrumentación de la política monetaria ha conseguido mayor efectividad.

En México, la política monetaria ha evolucionado gradualmente hasta establecer la tasa de interés como el principal instrumento para conseguir las metas de inflación planteadas por el banco central. En particular, diferentes estudios han señalado la creciente efectividad que la política monetaria ha alcanzado, generalmente mediante un sesgo contractivo, en la estabilidad del nivel general de precios. Sin embargo, al igual que ocurre en los distintos estudios elaborados internacionalmente, la perspectiva de la banca central es predominantemente macroeconómica, sin que haya evidencia de considerar explícitamente el desempeño económico sectorial en la implementación de la política monetaria. Al igual que establece la literatura internacional previa, conocer con mayor precisión el efecto de la política en la actividad económica sectorial, en particular la manufacturera, permitirá determinar de manera específica las fuentes por las que el canal de transmisión de las variaciones de la política monetaria resultaría potencialmente más efectivo. Adicionalmente, la posibilidad de encontrar efectos diferenciados en la actividad industrial derivados de los cambios fabri- 
cados por la banca central servirá para proveer más información que favorezca una mejor sincronización con las políticas de tipo fiscal.

Por su parte, el sector manufacturero mexicano ha atravesado por diferentes etapas de desarrollo. Sin embargo, diversas investigaciones han enfatizado en su importancia económica con relación a su contribución al progreso económico de largo plazo y el empleo (De Jesús, 2019; Carbajal y De Jesús, 2017), y a través de las externalidades positivas que surgen a partir de los encadenamientos productivos con el resto de los sectores económicos. No obstante, existe poca evidencia en México acerca de su respuesta ante los cambios en las medidas de política monetaria (Torres, 2017); particularmente se desconoce si estas medidas conducirían a efectos sectoriales diferenciados y, en su caso, de qué magnitud.

En este sentido, el objetivo de esta investigación consiste en analizar el efecto que un cambio permanente de tipo contractivo en la política monetaria tendría en las actividades manufactureras en México. En particular, se pretende estudiar si un choque unitario que eleve la tasa de interés de corto plazo tendría efectos diferenciados en la actividad manufacturera del país. Al respecto, las preguntas que se pretenden responder son: ¿Un choque contractivo permanente a la tasa de interés inducido por el banco central provocaría efectos diferenciados en el nivel de precios y la producción en los distintos sectores manufactureros? ¿Cuáles sectores resultarían más sensibles ante una variación común en la política monetaria?

Para responder estas cuestiones se implementa un modelo vectorial autorregresivo estructural en panel, conforme a la propuesta metodológica de Pedroni (2013). Esta metodología es adecuada para nuestros fines porque permite modelar la heterogeneidad inherente a las diferentes actividades manufactureras en el país, además de modelar el efecto de un choque común a la política monetaria, rasgo que resultaría más adecuado que un choque de tipo específico o idiosincrático, toda vez que el sector manufacturero en su conjunto comparte un mismo régimen de política monetaria en el país.

La investigación se organiza de la siguiente manera: en la segunda sección se elabora una revisión crítica de la literatura económica previa; en la tercera sección se exploran las principales características de los 
sectores manufactureros del país y su vínculo con la política monetaria. En la cuarta sección se explica la metodología que se implementará, para más adelante realizar el análisis de la evidencia empírica, y finalmente, se presentan las conclusiones.

\section{REVISIÓN DE LA LITERATURA}

Bernanke y Gertler (1995) y Carlino y DeFina (1995) fueron los primeros autores en documentar los efectos de un shock de política monetaria a nivel desagregado, a partir de los componentes del gasto agregado (inversión y consumo), en el primer caso, y de las regiones de los Estados Unidos, en el segundo. Documentos más recientes (Ganley y Salmon, 1997; Hayo y Uhlenbrock 1999; Raddatz y Rigobon, 2003; Ibrahim y Amin, 2005; Alam y Waheed 2006; Rodríguez y Padrón, 2008; Georgopoulos y Hejazi, 2009; Sengupta, 2014; Kutu y Ngalawa, 2016) se centran en identificar las respuestas en la producción sectorial originadas por un shock monetario no anticipado en diferentes países o regiones; además, en su mayoría emplean la técnica de Vectores Autorregresivos (VAR) o VAR estructurales. En general, sus resultados muestran que la manufactura y los bienes durables son las actividades más sensibles a los movimientos no anticipados de la política monetaria.

De manera particular, Ganley y Salmon (1997) analizan las respuestas de 24 sectores de la economía en el Reino Unido. Estiman un VAR distinto para cada sector e identifican que los sectores más sensibles frente a la política monetaria son la construcción y la manufactura (en especial, las pequeñas empresas); por el contrario, el sector menos sensible es la agricultura. En el caso de España, Rodríguez y Padrón (2008) identifican a la minería y la industria pesada (maquinaria y equipo mecánico) como los más afectados, mientras que en Canadá (Georgopoulos y Hejazi, 2009) e India (Sengupta, 2014) la industria más sensible es la manufactura. Los resultados de Raddatz y Rigobon (2003) muestran que en Estados Unidos el consumo de durables y no durables e inversión residencial son los de mayor respuesta a la política monetaria (PM), además observan que un choque al sector de alta tecnología tendría efectos significativos en la producción agregada. Hayo y Uhlenbrock (1999) muestran que en Alemania los metales no ferrosos, química, hierro, acero, electricidad y fabricación de maquinaria de oficina son los sectores que evidencian una mayor reducción en 
la producción en respuesta a un incremento no anticipado en la tasa de interés. En países de la OCDE el sector más afectado es el de la producción de bienes duraderos, con un mayor requerimiento financiero para producir y con una pequeña capacidad de endeudamiento (Dedola y Lippi (2005). En el caso de Pakistan, Alam y Waheed (2006) documentan a la minería, la manufactura, el comercio, así como finanzas y seguros como los de mayor respuesta. Por su parte, Kutu y Ngalawa (2016) encuentran que las autoridades monetarias de Sudáfrica tendrían poca influencia en el desempeño de la producción industrial en este país mediante el canal de tasas de interés, pero mayor influencia a través del canal del dinero.

Otros documentos en los que se analiza de manera específica a la manufactura son los de Gertler y Gilchrist (1994) e Ibrahim y Amin (2005). El primero estudia el mecanismo de transmisión de la política monetaria condicionado al acceso de las empresas a los mercados de capital en Estados Unidos. Sus resultados revelan que ante un choque monetario contractivo, las empresas manufactureras pequeñas se contraen en mayor medida que las grandes, y contribuyen hasta con $60 \%$ de la caída en el sector manufacturero total. En el segundo, mediante un modelo VCE estiman funciones de impulso-respuesta generalizadas para trazar la dinámica de la producción manufacturera agregada de Malasia ante un choque que aumente la tasa de interés de corto plazo. Sus resultados indican que la producción manufacturera disminuye en mayor medida que la producción agregada, incluso en presencia del canal de transmisión del tipo de cambio.

La respuesta diferenciada sectorial a la política monetaria depende, entre otros factores, de la intensidad del capital, la sensibilidad de la tasa de interés, el grado de apertura económica, sus estrategias de planeación de producción, la mayor efectividad del canal de transmisión monetaria (Sengupta, 2014), el tamaño de las empresas, sus características financieras (capacidad de endeudamiento y acceso al crédito) (Vespignani, 2012; Dedola y Lippi, 2005, respectivamente) vinculadas con la fortaleza del canal del costo del capital (Georgopoulos y Hejazi, 2009), así como la durabilidad de los bienes producidos (Peersman y Smets, 2002). 
Para el caso de América Latina, Quintero (2017) analiza, mediante un modelo autorregresivo de rezagos distribuidos (ARDL), la producción industrial de cinco países (Brasil, Chile, Colombia, Perú y México). Sus resultados muestran que un incremento inesperado en la tasa de interés genera un gran descenso de la producción industrial en México, Colombia y Perú, no así en Chile y Brasil. El origen de esta heterogeneidad en los resultados se debe al tipo de bienes producidos (de capital y de consumo durables) en los tres primeros, debido a que suelen ser más sensibles a la tasa de interés.

Bravo et al. (2003) utilizan un VAR para estudiar el efecto de la política monetaria en cuatro sectores de Chile (agricultura, industria, comercio y construcción); los más sensibles a la contracción monetaria son la construcción y el comercio, mientras que los que presentan un mayor impacto sobre la producción agregada son el comercio y la industria. En cuanto a los componentes del sector industrial (bienes de capital, bienes de consumo, bienes de exportación y bienes intermedios), el de bienes de capital es el más sensible, y los que más aportan a la caída de la producción industrial son los de bienes de capital y los de consumo.

\section{POLÍTICA MONETARIA Y SUBSECTORES MANUFACTUREROS EN MÉXICO}

En esta sección presentamos las características principales de la política monetaria en México con énfasis en el periodo 2010-2017, en el que sobresalen tres etapas distintivas, así como del sector manufacturero y sus diferentes ramas. Con respecto a la política monetaria en México, a partir de la independencia monetaria que se le otorgó en 1993, el Banco de México (Banxico) ha podido mantener la estabilidad de precios para beneficiar al crecimiento económico sostenido del país. De manera breve, en 1995 el Banxico fijó a la inflación como su objetivo principal, pero en 2003 ese objetivo se modificó a una meta anual de 3\% con un intervalo de variación en los precios de $\pm 1 \%$ (OECD, 2019). Una postura central del Banxico es que toma al exceso de dinero como un determinante de la inflación en el largo plazo; en otras palabras, si se decide aumentar la oferta monetaria más allá de la demanda, ello favorecerá al incremento de precios y, en consecuencia, la inflación (Medina y Laguna, 2016). 
Los objetivos operacionales del banco central se modificaron a lo largo del tiempo. Se pueden distinguir dos momentos sobresalientes. Durante el periodo de septiembre de 1995 a enero de 2008, su objetivo se basó en el conocido "corto", que se refiere mantener saldos agregados negativos; una vez implementado, el Banxico podía inyectar al sistema financiero la liquidez necesaria, aunque una parte de ella se ofrecía a tasas de interés punitivas (García et al., 2019). En un principio, el llamado corto se basaba en saldos acumulados al final del mes, pero de 2003 a 2008 se aplicó a los saldos acumulados al final del día. Por otro lado, la actual política monetaria, iniciada en enero de 2008, se lleva a cabo a través de una tasa objetivo para las operaciones interbancarias efectuadas durante la noche. Asimismo, desde el 26 de agosto de 2005 los anuncios de política monetaria del instituto central ya incluían las tasas que consideraban consistentes con los niveles prevalecientes del corto (Garcia et al., 2019). En la Gráfica 1 se puede observar la postura de la política monetaria basada en las tasas objetivo mencionadas.

GRÁFICA 1

POSTURA DE LA POLÍTICA MONETARIA MEXICANA

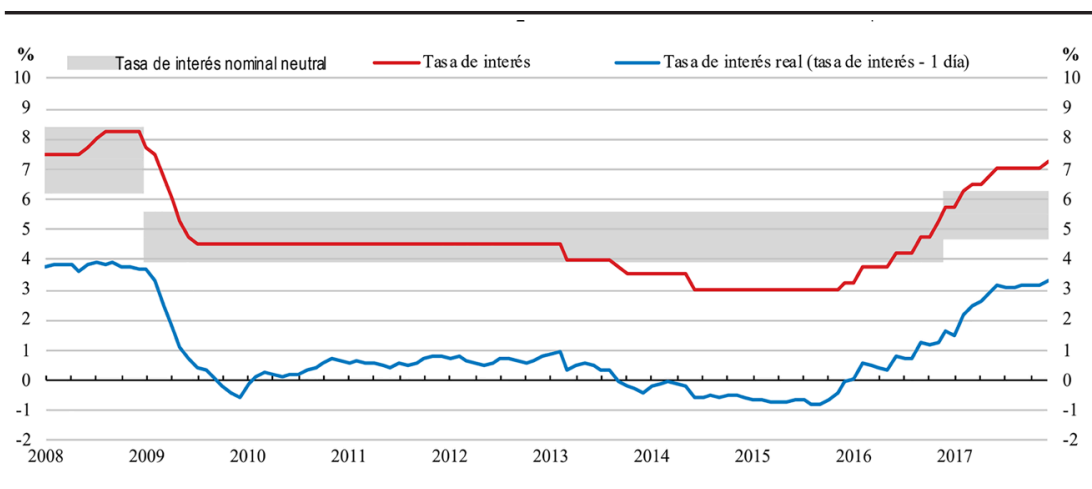

Nota: La tasa de interés real (ex ante) se calcula como la tasa de interés menos las expectativas de inflación para los siguientes 12 meses. El área gris representa el rango de la tasa de interés nominal neutral. Fuente: elaborado con datos de OECD, 2019.

En general, se distinguen tres periodos en la conducción de la política monetaria: en el primer periodo, de 2008 al primer trimestre de 2009, el banco central mantuvo las tasas de interés en los mismos niveles, pero a partir de la crisis financiera internacional de 2008 la tasa de interés disminuyó a 4.5\%, desde finales de 2009 hasta el primer trimestre de 2013, 
por lo que la política monetaria fue expansionista y estuvo asociada a un ambiente de inflación baja y estable. Como afirma Ibarra (2016), se fundamentó con la idea de que una política monetaria relajada provocaría una mayor disponibilidad de crédito para familias y empresas, para así incidir positivamente en el crecimiento de las actividades productivas. Por otro lado, Banxico continuó con una política más relajada desde 2013 hasta finales de 2015, cuando la tasa de interés llegó a ubicarse en 3\% y la tasa de interés real fue negativa (Gráfica 1). A partir de 2016, algunos sucesos internacionales complicaron las medidas de política monetaria, porque comprometían la estabilidad de precios y el crecimiento económico del país (OECD, 2017). La economía mundial comenzó a debilitarse, lo mismo que el comercio internacional y las inversiones. Por si fuera poco, las elecciones en Estados Unidos y la llegada del nuevo presidente y sus políticas económicas y comerciales transmitieron mucha incertidumbre en México, lo que ocasionó que el mercado cambiario se desestabilizará fuertemente. Aunado a ello, la caída en los precios del petróleo presionó a los ingresos del gobierno federal y contribuyó a reducir las inversiones en el sector energético. Por las razones anteriores, el Banxico impuso una política monetaria restrictiva y la tasa de interés rebasó los niveles de 7\% a finales de 2017 , como se aprecia en la Gráfica 1.

\subsection{El sector manufacturero}

La manufactura es uno de los sectores que más impulsa a la economía mexicana en términos de producto y empleo formal e informal. Tan sólo en 2015 los diferentes sectores manufactureros aportaron 17.3\% del PIB del país y $26.4 \%$ del empleo formal; en 2013 contribuyó con $48.2 \%$ de la producción total, $29 \%$ del valor agregado censal bruto y $33.9 \%$ de las remuneraciones totales (Carbajal y Carrillo, 2017: 15). Por otro lado, durante el periodo 2010-2017, la economía mexicana tuvo tasas de crecimiento decrecientes, que en promedio fueron de $2.9 \%$ (Cuadro 1). Si bien en 2010 y 2011 las tasas ascendieron a poco más de 4\%, después de 2014 comenzaron a declinar. Varios factores ocasionaron las bajas tasas de crecimiento de la actividad productiva: el debilitamiento de la economía mundial, la desaceleración de la economía estadounidense, la política monetaria restrictiva de la Reserva Federal y la depreciación del peso (OECD, 2017). Es posible deducir que la industria manufacturera contribuyó positivamente al crecimiento del PIB real de la economía. 
En el Cuadro 1 también se ilustran las ramas que más aportaron al PIB, como son maquinaria y equipo ( $9.6 \%$ promedio anual), equipo de transporte $(8.5 \%)$, equipo de computación y otros (5.6\%). Por otro lado, algunas ramas tuvieron un comportamiento negativo, las que incluyen a los insumos textiles y acabados de textiles, curtido y acabado de cuero y piel, y la industria química.

\section{CUADRO 1}

TASAS DE CRECIMIENTO REALES DEL PIB Y DE LA INDUSTRIA MANUFACTURERA

\begin{tabular}{|c|c|c|c|c|c|c|c|c|c|}
\hline & 2010 & 2011 & 2012 & 2013 & 2014 & 2015 & 2016 & \multicolumn{2}{|c|}{2017 Promedio } \\
\hline Producto interno bruto & 4.1 & 4.1 & 3.1 & 1.2 & 3.4 & 2.7 & 3.3 & 1.6 & 2.9 \\
\hline Alimentos & 1.8 & 3.1 & 2.6 & 0.7 & -1.0 & 2.5 & 4.5 & 1.5 & 2.0 \\
\hline Bebidas y tabaco & -0.2 & 1.7 & 0.2 & 1.7 & 4.7 & 9.5 & 6.0 & 2.1 & 3.2 \\
\hline Insumos textiles y aca bado de textiles & -2.5 & -7.3 & 4.5 & -7.1 & 1.3 & 2.8 & -3.4 & -1.1 & -1.6 \\
\hline Fabricación de productos textiles & -2.9 & -3.1 & 0.6 & -7.2 & 17.8 & 2.9 & 0.6 & -4.1 & 0.6 \\
\hline Fabricación de prendas de vestir & -0.1 & 0.3 & 1.3 & 4.1 & -4.4 & 9.1 & -5.2 & -1.0 & 0.5 \\
\hline Curtido y acabado de cuero $y$ piel & -1.0 & -2.6 & 1.5 & 1.1 & 0.3 & 1.4 & -0.6 & -1.7 & -0.2 \\
\hline Madera & 9.3 & -1.8 & 23.0 & -6.3 & -0.4 & 0.9 & -2.5 & -1.0 & 2.6 \\
\hline Papel & 1.5 & -1.1 & 5.8 & 2.7 & 4.2 & 2.8 & 2.5 & 1.1 & 2.4 \\
\hline Químicos & -1.1 & -0.9 & -3.6 & 2.7 & -1.1 & -6.7 & -3.3 & -2.7 & -2.1 \\
\hline Plástico y hule & 12.6 & 5.3 & -0.5 & -5.3 & 5.1 & 4.8 & -0.2 & 2.5 & 3.0 \\
\hline Productos minerales no metálicos & 7.9 & 2.2 & -0.8 & -3.0 & 6.0 & 3.7 & 0.8 & 2.2 & 2.4 \\
\hline Industrias metálicas básicas & -0.2 & 2.7 & -3.7 & 3.8 & 9.0 & -8.0 & 8.1 & -0.9 & 1.3 \\
\hline Productos metálicos & 10.2 & 6.7 & -2.6 & -10.0 & 9.9 & 3.3 & 1.9 & -1.4 & 2.3 \\
\hline Maquinaria y equipo & 60.2 & 8.4 & -6.3 & -5.0 & 9.9 & -1.9 & 2.6 & 8.6 & 9.6 \\
\hline Equipo de computación, comunicación, etc. & -1.1 & 5.6 & 0.3 & 9.4 & 17.0 & 6.5 & 4.9 & 2.5 & 5.6 \\
\hline Accesorios, aparatos eléctricos y equipo & -1.3 & -0.9 & 1.0 & -4.9 & 11.3 & 4.7 & 4.8 & -3.3 & 1.4 \\
\hline Equipo de transporte & 16.0 & 8.9 & 9.9 & 5.4 & 12.5 & 5.0 & 3.3 & 7.2 & 8.5 \\
\hline Muebles, colchones y persianas & -1.3 & 21.7 & -13.1 & 3.7 & 0.8 & -5.2 & -3.8 & 0.6 & 0.4 \\
\hline Otras industrias & 4.6 & 3.9 & 2.4 & 3.2 & 1.9 & 7.8 & 10.5 & 7.8 & 5.3 \\
\hline
\end{tabular}

Fuente: elaborado con datos del INEGI.

Una deducción de la segunda sección es que la industria manufacturera es sensible a los cambios no anticipados de la política monetaria. En particular, Quintero (2017) demuestra que en México el PIB posee una alta respuesta a los movimientos inesperados en la tasa de interés (un incremento de $1 \%$ en la tasa hace que la producción industrial disminuya significativamente), dada la importancia de la primera en el canal de transmisión de la política monetaria. En términos de subsectores, los dedicados a bienes de consumo duraderos y bienes de capital responden más a la tasa de interés que los sectores de bienes intermedios y de consumo no duradero. En la Gráfica 2 se muestra la evolución de la tasa de interés nominal (rezagada un año) y el crecimiento promedio de la producción de bienes duraderos y de capital, y no duraderos. En general, los datos indican una marcada relación inversa entre la producción y la tasa de interés establecida por el Banco de México a lo largo de 2010 a 2015. Es interesante observar que después de 2014 la caída en la tasa de interés impulsó a la producción manufacturera, tanto de bienes duraderos y de capital, así como los no duraderos. 
GRÁFICA 2

TASA DE INTERÉS NOMINAL Y PRODUCCIÓN MANUFACTURERA

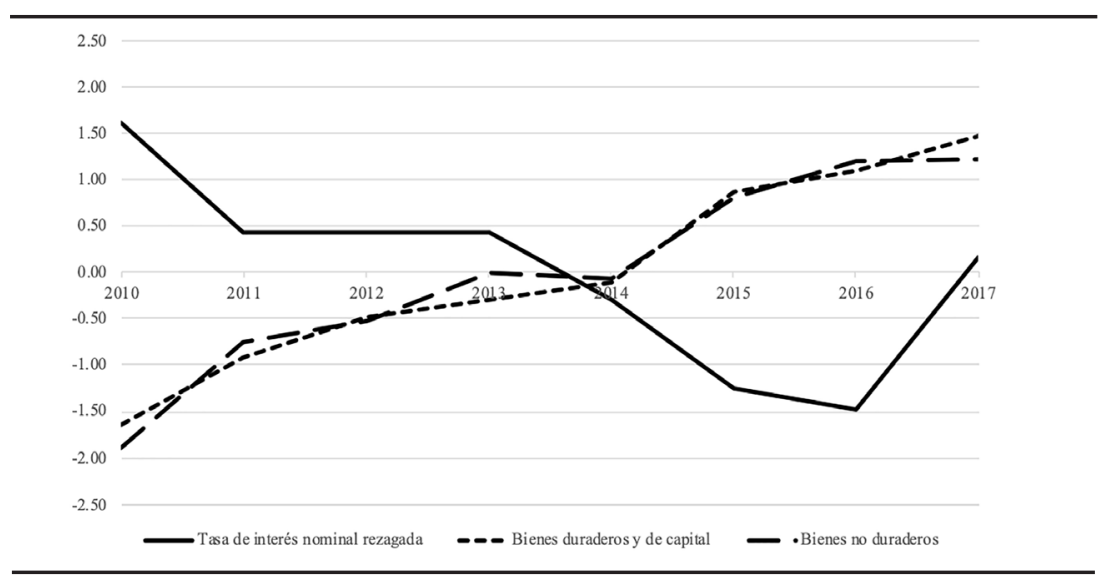

Fuente: elaborado con datos del INEGI.

En síntesis, la política monetaria mexicana ha cumplido con su objetivo de controlar la inflación por medio de la tasa de interés, principal canal de transmisión. Asimismo, la exploración inicial de la asociación entre la producción manufacturera y la tasa de interés señala que la primera responde con fuerza a la política monetaria.

\section{Aspectos Metodológicos}

Con la finalidad de investigar el efecto que un cambio sorpresivo de tipo contractivo en la política monetaria tendría sobre el comportamiento del nivel de producción y precios en los subsectores manufactureros, se implementa un modelo estructural de vectores autorregresivos en panel conforme a la propuesta de Pedroni (2013). Esta metodología es adecuada para nuestros fines porque permite incorporar la heterogeneidad en el desempeño al interior del sector manufacturero. Adicionalmente, este enfoque ofrece la posibilidad de establecer restricciones que facilitan la identificación y estimación de los choques estructurales a la política monetaria y su efecto sobre las variables endógenas.

En particular, si partimos de la siguiente representación de media móvil para un panel VAR en forma reducida: 


$$
\Delta \mathbf{z}_{i t}=\mathbf{F}_{i}(L) \mathbf{u}_{i t}
$$

sustituimos la relación entre los choques en forma reducida y estructural conforme a la igualdad $\mathbf{u}_{i t}=\mathbf{B}_{i}^{-1} \boldsymbol{\epsilon}_{i t}$ y se obtiene la siguiente representación en forma estructural:

$$
\Delta \mathbf{z}_{i t}=\mathbf{A}_{i}(L) \boldsymbol{\epsilon}_{i t}
$$

En este caso, la matriz $\mathbf{A}_{\mathrm{i}}$ (L) recoge las funciones de impulso respuesta correspondientes al choque estructural $\boldsymbol{\epsilon}_{\mathrm{it}}$. Pedroni (2013) establece que el choque estructural $\epsilon_{\text {it }}$ está compuesto por la suma de un choque determinado común $\overline{\boldsymbol{\epsilon}}_{\mathrm{t}}$, y otro denominado idiosincrático o específico $\tilde{\boldsymbol{\epsilon}}_{\mathrm{it}}$, de tal forma que ambos tipos de choque también pueden identificarse y estimarse. Para lograrlo, sugiere utilizar una representación de factores comunes, de manera que su descomposición se expresa como $\boldsymbol{\epsilon}_{\mathrm{it}}=\Lambda_{\mathrm{i}} \overline{\boldsymbol{\epsilon}}_{\mathrm{t}}+\tilde{\boldsymbol{\epsilon}}_{\mathrm{it}}$, donde $\Lambda_{\mathrm{i}}$ es una matriz diagonal de tamaño $M \times M$, donde cada elemento representa las cargas específicas $\lambda_{\mathrm{i}, \mathrm{m}}, m=1, \ldots M$ asociada a los choques comunes, y adicionalmente suponemos que ambos tipos de choques son ortogonales entre sí.

Los choques estructurales, en su representación de media móvil, pueden recuperarse mediante su relación con los choques en forma reducida, tal como se describe en la expresión $\mathbf{u}_{\mathrm{it}}=\mathbf{A}_{\mathrm{i}}(0) \mathbf{\epsilon}_{\mathrm{it}}$, mientras que la relación $\mathbf{F}_{\mathrm{i}}(L) \mathbf{A}_{\mathrm{i}}(0)=\mathbf{A}_{\mathrm{i}}(L)$ permite obtener las funciones de impulso respuesta estructurales $\mathbf{A}_{\mathrm{i}}(L)$. Al respecto, es importante mencionar que $\mathbf{A}_{\mathrm{i}}(0)$ representa las funciones de impulso respuesta estructurales contemporáneas e incorpora las restricciones estructurales relevantes para identificar, y posteriormente estimar, los choques y respuestas estructurales para cada una de las unidades bajo análisis.

La propuesta de Pedroni (2013) para descomponer los choques estructurales compuestos, $\boldsymbol{\epsilon}_{\mathrm{it}}$, y sus respectivas funciones de impulso respuesta, en un componente común, $\overline{\boldsymbol{\epsilon}}_{\mathrm{it}}$, y otro específico, $\tilde{\boldsymbol{\epsilon}}_{\mathrm{it}}$, es relevante porque permite, en el primer caso, disponer de una aproximación al hecho de que la política monetaria es común para el conjunto 
de los subsectores manufactureros mexicanos. Por su parte, el componente específico permitiría conocer aquellos choques que inducirían variaciones en el comportamiento de los subsectores manufactureros pero vinculados a las características y condiciones específicas a cada subsector.

Al respecto, Pedroni (2013) se basa en la presencia efectos de tiempo para describir los componentes comunes entre las variables que conforman el vector autorregresivo, calculada mediante $\Delta \overline{\mathbf{z}}_{\mathrm{t}}=N^{-1}$ $\sum_{i=1}^{N} \Delta \mathbf{z}_{\mathrm{it}}$, donde $\Delta \mathbf{z}_{\mathrm{it}}$ es el vector de variables endógenas del modelo en primera diferencia. Análogamente a la identificación y estimación de los choques estructurales compuestos, el autor define la representación estructural del VAR con las variables endógenas en términos de la presencia de los efectos de tiempo en su forma de media móvil mediante $\Delta \overline{\mathbf{z}}=\overline{\mathbf{A}}(L) \overline{\mathbf{\epsilon}}$, donde los choques en forma reducida y estructurales están vinculados mediante la expresión $\overline{\mathbf{u}}_{\mathrm{t}}=\overline{\mathbf{A}}(0) \overline{\boldsymbol{\epsilon}}_{\mathrm{t}}$, y en el caso de las funciones de impulso respuesta comunes mediante $\overline{\mathrm{F}}(L) \overline{\mathbf{A}}(0)=\overline{\mathbf{A}}(L)$.

\subsection{Especificación del modelo VAR}

El modelo que se implementa para investigar el probable impacto de las variaciones no anticipadas en la política monetaria en el nivel de precios y producción en la actividad sectorial industrial en México es un vector autoregresivo estructural en panel que incorpora tres variables endógenas para los 21 subsectores manufactureros. La identificación y estimación de los choques a la política monetaria y las funciones de impulso respuesta asociadas siguen un enfoque recursivo, conforme a la propuesta de Christiano et al. (1996) y Christiano et al. (1998a), entre otros, en la literatura macroeconómica. En este enfoque recursivo, el banco central tiene la posibilidad de acceder a información económica relevante de forma contemporánea para establecer los cambios que ocurrirán en el instrumento de política monetaria, en tanto las variables económicas son ortogonales a las variaciones contemporáneas en los cambios de la política monetaria, de tal forma que su efecto ocurriría, pero con un retardo en el tiempo.

En esta investigación se utiliza la tasa de interés de corto plazo como el instrumento a través del cual las variaciones en la política monetaria, instrumentadas por el banco central en México, inciden sobre los precios y producción de los subsectores manufactureros. Esta elección obedece a que es el instrumento que el Banco de México ha implemen- 
tado para incidir indirectamente en la formación de precios a través del tiempo. En particular, las restricciones para identificar los choques comunes a la política monetaria a partir del supuesto de recursividad se expresan en la siguiente matriz de impactos contemporáneos:

$$
\overline{\mathbf{A}}(0)=\left[\begin{array}{ccc}
\bar{a}_{11} & \ldots & \vdots \\
\bar{a}_{21} & \bar{a}_{22} & \vdots \\
\bar{a}_{31} & \bar{a}_{32} & \bar{a}_{33}
\end{array}\right]
$$

Al respecto, el orden de las variables endógenas especificado en el vector de efectos comunes de tiempo en primeras diferencias es $\Delta \overline{\mathbf{z}}_{\mathrm{t}}=\left[\Delta \overline{P m}_{t} \Delta \overline{Y m}_{t} \Delta \overline{I n t}\right]$, donde $\overline{P m}_{t}$ y $\overline{Y m}_{t}$ significan el promedio del nivel de precios y producción de los 21 subsectores manufactureros, respectivamente. Con relación a la tasa de interés, el promedio corresponde a su valor original debido a su alcance nacional, de tal forma que $\overline{I_{n}}{ }_{t}=$ Int $_{t}$. En el tercer renglón de la matriz de impactos contemporáneos se establece una relación que se acerca a una regla de retroalimentación del Banco de México, en la que suponemos que la autoridad monetaria establece la tasa de interés a partir de la evaluación de las condiciones económicas contemporáneas, en este caso para los precios y producción industriales. Este supuesto es adecuado si se considera que el banco central tiene acceso a información económica a cualquier nivel de desagregación, inclusive sectorial, para establecer el curso que seguirá su instrumento de política monetaria. Adicionalmente, en el primer renglón se fundamenta que una variación en el precio de un subsector en particular puede incidir contemporáneamente, por ejemplo, en el mismo mes en su ajuste, en tanto que las variaciones en la producción y la propia política monetaria lo harían, pero con un retraso. Esto implica que los agentes económicos ajustan los precios antes de observar algún cambio en la producción o en la conducción de la política monetaria. Por su parte, la producción se ajustaría contemporáneamente ante cambios en los precios y la propia actividad manufacturera, mientras que reaccionaría con un retardo ante las variaciones de la política monetaria. Este aspecto implica que los agentes económicos establecen su producción previamente a la observación de los ajustes en la política monetaria, generalmente en periodos posteriores al diseño de los planes de producción y, por otro lado, el retraso en el ajuste considera que los agentes 
económicos no se ajustan contemporáneamente porque es altamente plausible que encuentren costoso modificar sus planes de producción de forma inmediata.

\subsection{Descripción de los datos}

Se utilizaron datos mensuales para los 21 subsectores manufactureros, cuya clasificación obedece a la propuesta por el SCIAN. La producción manufacturera para cada sector se midió a través del Índice del Volumen Físico que publica el INEGI con información de junio de 2010 a 2017. Para el caso de los precios, estos provienen de los índices de precios al productor para cada subsector manufacturero, también publicados por el INEGI, con la misma periodicidad. La tasa de interés de corto plazo corresponde a los CETES a 29 días. Los datos fueron transformados a logaritmos, con excepción de la tasa de interés. En tal sentido, el panel se conforma por 21 unidades de sección cruzada y 84 realizaciones en el tiempo, lo que da un total de 1764 observaciones en conjunto.

Con la finalidad de corroborar si las variables endógenas en el vector $\Delta \mathrm{z}_{\mathrm{it}}$ tienen un comportamiento estocástico estacionario, se aplicó, en primera instancia, la prueba de raíz unitaria en panel propuesta por Im, Pesaran y Shin (IPS) (2003), ${ }^{2}$ que considera la heterogeneidad entre las unidades de sección cruzada, las variables de precios y producción. En el Cuadro 2 se presentan los resultados de esta prueba; se puede apreciar que existe evidencia para no rechazar la hipótesis nula acerca de la presencia de raíz unitaria en el nivel de precios $\left(P m_{i t}\right)$ y producción $\left(Y m_{i t}\right)$ en los 21 subsectores manufactureros. Por su parte, la prueba indica que es posible rechazar la misma hipótesis nula para las variables en primeras diferencias, y sugiere que son integradas de orden 1, I(1). Adicionalmente, la prueba Dickey-Fuller aplicada a la tasa de interés $\left(\right.$ Int $t_{t}$ indica que la tasa de interés puede considerarse integrada de orden 1.

2 El estadístico W de la prueba IPS corresponde al estadístico t-barra cuando se incorporan suficientes retardos para considerar la correlación serial, tal como en una prueba Dickey-Fuller aumentada (DFA). De acuerdo con Im, Pesaran y Shin (2003), las propiedades para muestras finitas del estadístico son adecuadas, inclusive se desempeñan mejor que en pruebas basadas en estadísticos de verosimilitud. De hecho, el número de periodos relativamente amplio en la muestra $(\mathrm{T}=84)$ permitiría realizar las pruebas de raíz unitaria a las series individuales. 
CUADRO 2

PRUEBAS DE RAÍZ UNITARIA IPS EN PANEL Y DF AUMENTADA PARA LAS VARIABLES DEL MODELO

\begin{tabular}{cccc}
\hline Variable & Estadístico-W & Prob. & Rezago máximo \\
$P m_{i t}$ & 9.27761 & 0.98 & 11 \\
$\Delta P m_{i t}$ & -9.29339 & 0.00 & 11 \\
$Y m_{i t}$ & 5.09902 & 0.98 & 11 \\
$\Delta Y m_{i t}$ & -19.9719 & 0.00 & 11 \\
$V$ Variable & Estadístico DF & Prob. & 3 \\
\hline Int & 0.1172 & 0.98 & 2 \\
\hline Int & -3.0768 & 0.00 & 3 \\
\hline
\end{tabular}

Nota: se aplicó complementariamente, a la tasa de interés, la prueba de Lee y Strazicich (2013) que considera posibles cambios estructurales en las series. Los resultados, que corroboran el mismo orden de integración obtenido a partir de la prueba DF aumentada, no se reportan aquí pero se encuentran a disposición bajo requerimiento.

Fuente: elaborado con estimaciones propias.

\section{EVIDENCIA EMPÍRICA}

En la Gráfica 3 se describe la contribución de cada choque en la descomposición de varianza para los niveles de precios y producción distribuida en cuartiles. Se aprecia que la contribución del choque común directo al nivel de precios disminuye continuamente para las industrias manufactureras ubicadas en los diferentes cuartiles; sin embargo, esta contribución a la variación aumenta gradualmente en el caso de los choques comunes a la producción y la tasa de interés de corto plazo. Este último caso es relevante, debido a que sugiere que los movimientos no anticipados permanentes en la conducción de la política monetaria tendrían efectos permanentes en el nivel de precios, preservando su importancia como instrumento estabilizador de la inflación. 


\section{GRÁFICA 3}

\section{DESCOMPOSICIÓN DE VARIANZA PARA LOS CHOQUES COMUNES}

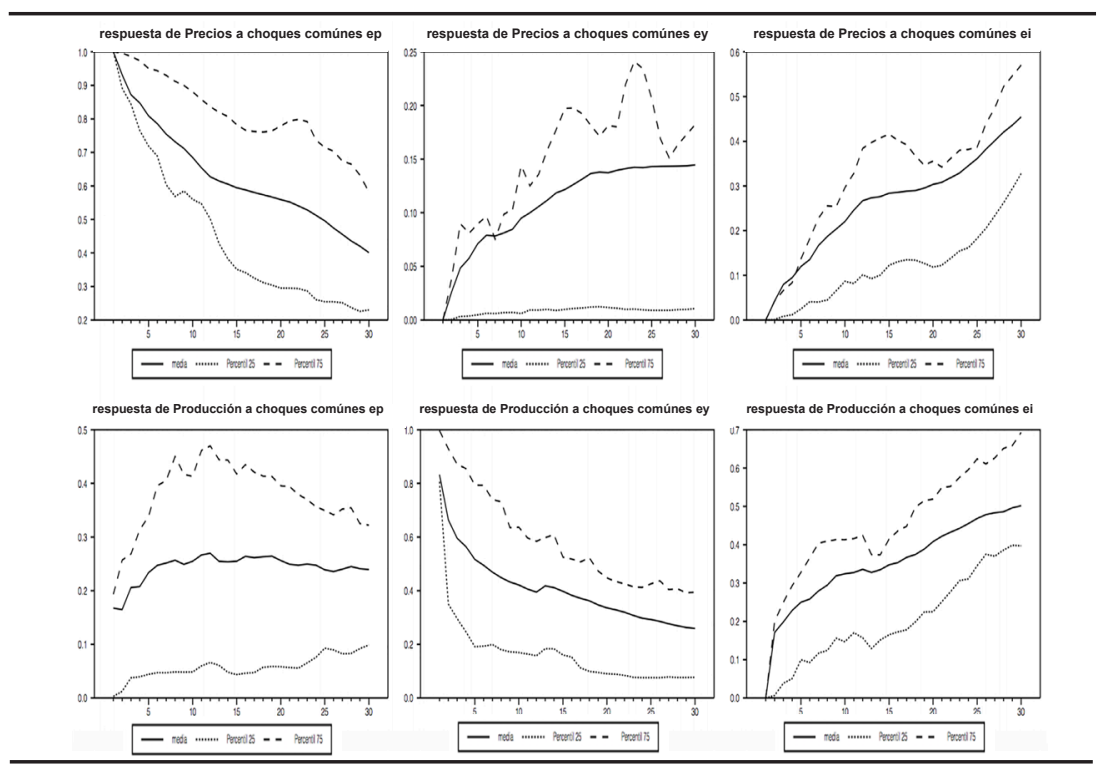

Fuente: elaborado con estimaciones propias.

Una situación similar se observa con la descomposición de la varianza para los choques comunes sobre el nivel de producción. Particularmente, la contribución de los choques comunes de precios hacia la producción parece llegar a su máximo aproximadamente a los 12 meses, para posteriormente disminuir lentamente. Este proceso es similar en el caso de la contribución de los choques directos a la producción, aunque ocurre con mayor rapidez. Esta situación implica que los choques a la política monetaria ganarían relevancia con el tiempo, indicando así que un efecto de no-neutralidad sobre la producción industrial en los distintos sectores manufactureros estaría presente. En este sentido, la descomposición de la varianza indica que la política monetaria puede ser efectiva en el corto y largo plazo, tanto en la estabilización de precios como en su efecto sobre la producción industrial.

Las funciones de impulso respuesta de los niveles de precios y producción a los choques comunes en el modelo panel VAR estructural para los distintos subsectores manufactureros se muestran distribuidas en cuartiles en la Gráfica 3. Un primer rasgo que sobresale es la reacción heterogénea de las distintas industrias manufactureras a los diferentes 
choques comunes, en particular respecto al choque permanente dirigido a la política monetaria. Este resultado indica que los agentes económicos en la industria manufacturera adaptan sus estrategias de precios y planes de producción de forma diferenciada en respuesta a los cambios permanentes en la tasa de interés inducidos por el banco central. Un caso lo constituyen los agentes económicos ubicados en el cuartil 25, que responden ajustando sus precios gradualmente, aunque con menor sensibilidad a variaciones de tipo contractivo en la política monetaria. Por ejemplo, las industrias en este cuartil inicialmente parecen no responder significativamente durante los primeros seis meses, posteriormente, se observa un ligero aumento en los precios alrededor de $0.5 \%$, que se desvanece aproximadamente en año y medio, para enseguida comenzar un declive en el nivel de precios que alcanzaría $0.4 \%$ aproximadamente dos años y medio después.

El patrón que siguen las industrias ubicadas alrededor del promedio es similar al observado en las industrias en el primer cuartil, pero con algunas variaciones en la magnitud de respuesta. Este sentido, los subsectores inicialmente responden positivamente, aunque con una magnitud pequeña de alrededor de $0.2 \%$, que llega a alcanzar un punto de inflexión aproximadamente en un año, cuando iniciaría un declive en el nivel de precios para llegar un nivel $0.2 \%$ menor al inicial en el momento del choque, pero dos años y medio después. Por otro lado, un número de industrias parece ser menos sensible a los choques contractivos de política monetaria, ya que algunas de las ubicadas en el cuartil 75 observan una respuesta positiva, aunque apenas mayor a $0.1 \%$, de tal forma que los choques positivos a la tasa de interés tendrían efectos menos significativos en comparación con el resto de las industrias manufactureras.

Por su parte, el ajuste de la producción industrial ante los cambios permanentes no anticipados en la política monetaria ocurre más rápido que en el caso de los precios, aunque también con una marcada heterogeneidad. Por ejemplo, en el caso de las industrias ubicadas en primer cuartil, la producción se contrae hacia el segundo mes después del choque en una pequeña magnitud, aunque para el quinto mes alcanzaría una contracción acumulada cerca a $0.2 \%$. Después de algunos episodios de variabilidad, a partir del primer año se observa un declive permanente que alcanzaría una contracción cercana a $0.6 \%$, aproximadamente a los 30 meses. En el caso de las industrias alrededor de la 
respuesta promedio, el comportamiento es similar, aunque la producción disminuiría más lentamente. Al respecto, se aprecia que durante el primer año ocurren algunas contracciones; las más significativas son en el quinto y octavo mes; sin embargo, la producción industrial en estos subsectores iniciaría su declive año y medio después del choque a la tasa de interés para alcanzar una contracción alrededor de $0.3 \%$. Las industrias manufactureras ubicadas en el cuartil 75 han experimentado una respuesta positiva, aunque bastante modesta de aproximadamente $0.1 \%$, de tal forma que los movimientos en la tasa de interés parecen tener un efecto de neutralidad en estos subsectores.

El patrón de respuesta seguido por el nivel de precios y la producción en los distintos subsectores industriales ante un choque contractivo permanente en la política monetaria se apega al patrón observado previamente en estudios de tipo macroeconómico (Christiano et al., 1998), en el sentido que los agentes económicos ajustan los precios más lentamente hacia la baja, mientras que la producción tiende a contraerse con mayor rapidez. Desde el punto de vista metodológico, este resultado permite corroborar la viabilidad del modelo panel recursivo estimado. Empero, el efecto diferenciado de las variaciones en la tasa de interés sobre la actividad industria tiene implicaciones relevantes, ya que, si la política monetaria tiene un efecto no neutral en los subsectores manufactureros, éste se canaliza en mayor medida a través de algunos sectores más sensibles, que por tanto terminan absorbiendo mayormente el impacto contractivo. Es decir, la heterogeneidad observada significa que la efectividad de la política monetaria sobre el nivel de precios y la producción industriales parece descansar principalmente en algunas industrias.

Respecto a la respuesta en los niveles de precios como resultado de choques directos no anticipados a los propios precios, en la Gráfica 4 se aprecia un patrón de ajuste hacia el alza. Aunque éste es el esperado, debe notarse que también es heterogéneo; específicamente en el caso de las industrias en el primer cuartil, se observa un aumento modesto cercano a $0.05 \%$, que se mantiene estable en el tiempo. Por otro lado, en las industrias que experimentaron un alza similar al promedio, se percibe un aumento cercano a $0.14 \%$ en el mismo mes del impacto, para posteriormente evolucionar ligeramente hacia el alza. En el caso de los precios de los subsectores manufactureros ubicados en el cuartil 75 , el aumento en precios es más volátil, con $0.23 \%$ por encima de su 
nivel inicial en el primer mes; alcanza su pico en el mes 20 y siguen aumentos permanentes, aunque cada vez menores. Sobre la reacción del nivel de precios ante un choque que aumente la producción, se puede apreciar que las industrias ubicadas en el primer cuartil presentan un declive más rápido que aquéllas ubicadas en el efecto medio, donde el declive ocurre varios meses después. Sin embargo, los subsectores manufactureros ubicados en el tercer cuartil parecen menos sensibles a las variaciones no anticipadas de la política monetaria.

GRÁFICA 4

RESPUESTAS ESTIMADAS DEBIDO A LOS CHOQUES COMUNES

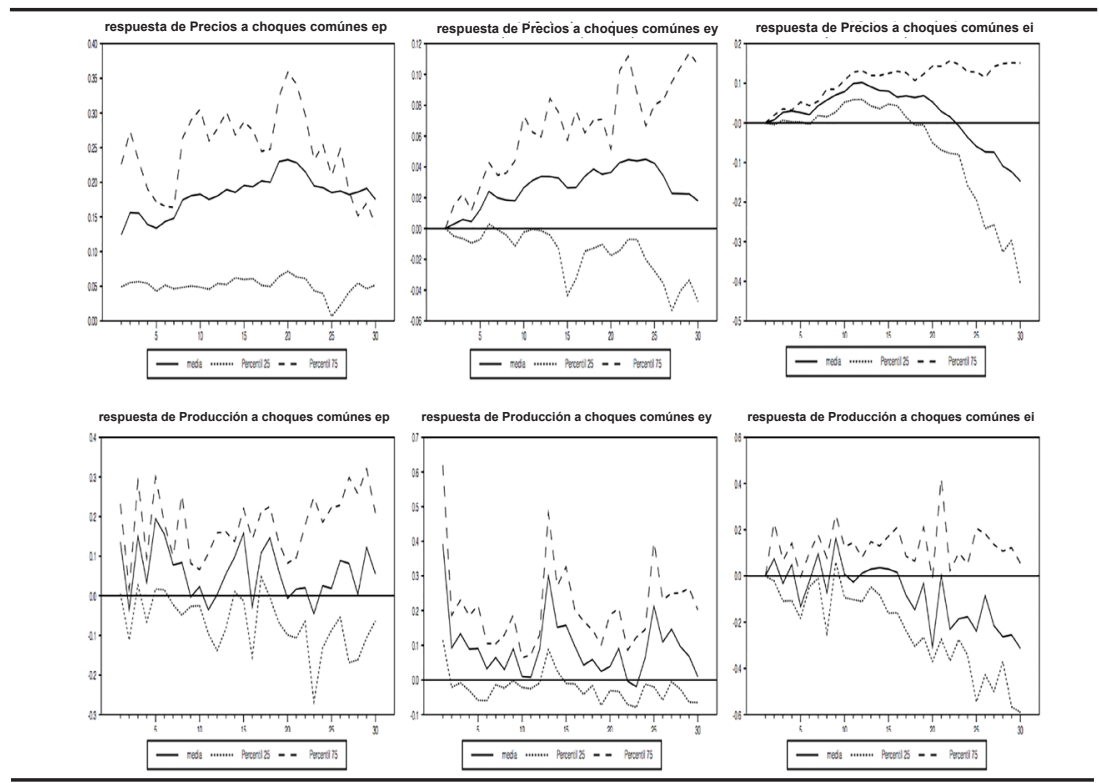

Fuente: elaborado con estimaciones propias.

Adicionalmente, una revisión de la respuesta individual de cada subsector industrial ante un choque permanente no anticipado en la política monetaria que eleve la tasa de interés permite observar de forma específica el efecto diferenciado. Al respecto, en el Cuadro 3 se aprecia que las industrias de productos textiles, la fabricación de equipo de cómputo y comunicaciones, y de equipo de transporte, tendrían en promedio un efecto contractivo mayor sobre los precios que el resto de los subsectores, mientras que algunas industrias presentan un efecto medio positivo más pequeño. Por su parte, 
el análisis de la respuesta de la producción industrial a las variaciones de la política monetaria indica que es en esta variable donde ocurriría el principal ajuste. Al respecto, los subsectores dedicados a la fabricación de productos textiles (314), de muebles, colchones y persianas (337), productos a base de minerales no metálicos (327) y de insumos y acabados textiles (313), experimentaron las contracciones más significativas. Este resultado sugiere que el efecto diferenciado de la política monetaria en la actividad industrial estaría asociado a las características estructurales específicas de cada industria, en cuyo caso, los canales de la tasa de interés y del crédito representan mecanismos de transmisión que ofrecen explicaciones plausibles acerca de las fuentes de estas respuestas industriales heterogéneas (Peersman y Smets, 2005; Dedola y Lippi, 2005).

Diversos autores, entre ellos Peersman y Smets (2005) y Dedola y Lippi (2005), señalan que la sensibilidad de la actividad industrial ante variaciones en la postura de la política monetaria puede trazarse a través del canal tradicional de la tasa de interés y del canal de crédito. Con relación al primero de estos canales de transmisión, los autores señalan que las industrias que producen bienes duraderos experimentan un efecto mayor en comparación con aquellas industrias que producen bienes no duraderos, debido a que su demanda de consumo o de inversión es más sensible a los cambios en la tasa de interés. Por su parte, con respecto al canal del crédito, la estructura financiera de la industria condicionaría la sensibilidad de la actividad industrial a los cambios en la política monetaria a través del canal de crédito. De esta manera, una política monetaria que conduzca a un alza en la tasa de interés no solamente aumentaría el costo de capital por esta vía, sino también se reduciría el colateral y el flujo de efectivo en las industrias, e induciría un aumento en el costo de financiamiento externo. En consecuencia, las industrias con mayor valor neto podrán acceder a créditos menos costosos y, por tanto, las variaciones en la política monetaria tendrían un menor efecto. 


\section{CUADRO 3}

IMPACTO MÁXIMO Y ACUMULADO PROMEDIO EN EL NIVEL DE PRECIOS Y PRODUCCIÓN RESULTADO DE UN CHOQUE PERMANENTE A LA TASA DE INTERÉS DE CORTO PLAZO

\begin{tabular}{|c|c|c|c|c|c|c|}
\hline Industria & $\begin{array}{l}\text { Promedio } \\
\text { acumulado } \\
\text { (Precios) }\end{array}$ & $\begin{array}{l}\text { Máximo } \\
\text { impacto }\end{array}$ & Mes & $\begin{array}{l}\text { Promedio } \\
\text { acumulado } \\
\text { (Producción) }\end{array}$ & $\begin{array}{l}\text { Máximo } \\
\text { impacto }\end{array}$ & Mes \\
\hline 311 & 0.08 & -0.01 & Siete & 0.1 & -0.1 & Cinco \\
\hline 312 & 0.08 & -0.01 & Tres & -0.09 & -0.59 & Treinta \\
\hline 313 & 0.1 & 0 & Uno & -0.22 & -0.83 & Treinta \\
\hline 314 & -0.11 & -0.76 & Treinta & -0.8 & -2.36 & Veintinueve \\
\hline 315 & 0.01 & -0.15 & Treinta & 0.29 & -0.53 & Cinco \\
\hline 316 & 0.1 & 0 & Uno & -0.07 & -1.02 & Veiticinco \\
\hline 321 & 0.08 & 0 & Uno & -0.09 & -0.29 & Ocho \\
\hline 322 & 0.08 & -0.01 & Dos & 0.02 & -0.07 & Cinco \\
\hline 323 & -0.01 & -0.05 & Nueve & -0.04 & -0.33 & Veinticuatro \\
\hline 324 & 0.36 & 0 & Uno & -0.19 & -0.53 & Ocho \\
\hline 325 & 0.15 & -0.13 & Seis & -0.19 & -0.5 & Veintisiete \\
\hline 326 & -0.07 & -0.41 & Treinta & 0.17 & -0.31 & Veintidos \\
\hline 327 & -0.04 & -0.22 & Veintiocho & -0.27 & -0.67 & Veintisiete \\
\hline 331 & 0.14 & -0.01 & Cuatro & 0.4 & -0.06 & Cinco \\
\hline 332 & 0.09 & -0.04 & Dos & -0.13 & -0.57 & Veintinueve \\
\hline 333 & -0.1 & -0.77 & Treinta & 0.06 & -0.73 & Cinco \\
\hline 334 & -0.32 & -1.34 & Treinta & 0.09 & -0.3 & Doce \\
\hline 335 & -0.01 & -0.28 & Treinta & -0.18 & -0.7 & Veintinueve \\
\hline 336 & -0.27 & -1.09 & Treinta & 0.4 & 0 & Uno \\
\hline 337 & 0.05 & -0.01 & Veintisiete & -0.68 & -2.53 & Veinte \\
\hline 339 & -0.01 & -0.43 & Treinta & -0.19 & -1.35 & Veintinueve \\
\hline
\end{tabular}

Los dígitos en la columna denominada industria, corresponden a la clasificación por subsectores de la industria manufacturera del SCIAN que utiliza el INEGI, como se describe enseguida: 311 Ind. Alimentaria; 312 Ind. Bebidas y tabaco; 313 Fab. de insumos y acabados textiles; 314 Fab. de productos textiles (excepto prendas de vestir); $315 \mathrm{Fab}$. de prendas de vestir; 316 Curtido y acabado de cuero y piel, fab. de productos de cuero, piel y sucedáneos; 321 Ind. de la madera; 322 Ind. del papel; 323 Impresión e ind. Conexas; 324 Fab. productos y derivados del petróleo y carbón; 325 Ind. química; 326 Ind. del plástico y del hule; 327 Fab. de prod. a base de miner. no metálicos; 331 Ind. metálicas básica; 332 Fab. de productos metálicos; 333 Fab. de maquinaria y equipo; 334 Fab. de equipo de computación y comunicaciones; 335 Fab. de eq. de gen. eléctrica y apar. eléctricos; 336 Equipo de transporte; 337 Fab. de muebles, colchones y persianas; 339 Otras ind. manufactureras.

Fuente: elaborado con estimaciones propias.

Con el propósito de profundizar en el análisis de las fuentes de la heterogeneidad observada en la respuesta permanente de la producción industrial en los subsectores manufactureros ante cambios permanentes sorpresivos en la política monetaria, en el Cuadro 4 se presentan los resultados de las estimaciones de cinco modelos cuya variable dependiente es el impacto acumulado promedio, en valor absoluto, en la producción de los 21 subsectores manufactureros. En correspondencia 
con el canal convencional de la tasa de interés se recurrió a una medida de intensidad de capital, calculada de acuerdo con Hayo y Uhlenbrock (1999) y Peersman y Smets (2005), como la razón entre la inversión realizada sobre el valor agregado (I/VA), para cada subsector manufacturero. Según estos autores, las industrias con mayor intensidad de capital tenderían a ser más sensibles a los cambios en el costo del capital, el cual también cambia con la tasa de interés, el instrumento de política monetaria.

Adicionalmente, en la literatura se considera que el gasto en bienes duraderos es más sensible a las variaciones en la tasa de interés que el gasto en bienes no duraderos. Para incorporar esta característica se incluyó una variable ficticia interactiva que toma un valor de 1 si el subsector produce bienes duraderos, y un valor de 0 en caso contrario. Similarmente, se construyó una variable ficticia para los subsectores que producen bienes no duraderos. Por su parte, en concordancia con el canal de crédito, se utilizó una variable que mide la proporción de micro y pequeños establecimientos (EMP) en cada uno de los subsectores. ${ }^{3}$ En este caso, debido a que las empresas con menor tamaño enfrentarían restricciones más severas para acceder a financiamiento mediante créditos bancarios, bursatilización e inclusive financiamiento proveniente del extranjero, se espera que sean más sensibles a los cambios en la tasa de interés que las empresas grandes. La información utilizada en la medición de las variables explicativas se obtuvo del Censo Económico 2014 del INEGI para los 21 subsectores.

Los resultados de las regresiones para el modelo 1, que incluye como regresores a las medidas globales de intensidad de capital (I/VA) y la proporción de micro y pequeños establecimientos (EMP), que abarcan los subsectores manufacturero, productores de bienes duraderos y no duraderos, muestran que individualmente ambas medidas son estadísticamente no significativas. La prueba F, por su parte, favorece la no significación estadística conjunta, lo que resulta en un pobre ajuste de la regresión. Se analizaron especificaciones alternativas para determinar

3 En la literatura se pueden encontrar medidas alternativas que permiten aproximar los costos de agencia diferenciales entre industrias, tales como medidas de apalancamiento, de estructura de vencimiento de deuda, y de cobertura; sin embargo, estas medidas se construyen a partir de información que proviene de hojas de balances que no se encuentran disponibles para los establecimientos en los 21 subsectores manufactureros en México. 
si el impacto heterogéneo de la política monetaria en la producción industrial puede ser explicado cuando se distingue entre la durabilidad de los bienes producidos. Los modelos 2 y 3 incluyen, además de las medidas globales de intensidad de capital y el tamaño de las empresas, dos variables interactivas que permiten distinguir las fuentes de la heterogeneidad cuando se consideran únicamente a los subsectores que producen bienes no duraderos. En el modelo 3 se añadió también una variable que mide la proporción de los establecimientos grandes $(\mathrm{G})$ en cada subsector. En el caso del modelo 2, las variables globales que miden el tamaño de los establecimientos (EMP) en el total de subsectores, y en aquéllos que producen bienes no duraderos (EMP no durables), aparecen con un signo positivo y una significación estadística de $10 \%$; sin embargo, el estadístico $\mathrm{F}$ indica la ausencia de significación estadística conjunta en el modelo, y un pobre ajuste con una R-cuadrada ajustada de 0.014. Estos resultados son similares en el modelo 3, en el que las empresas micro y pequeñas perdieron significación estadística.

Los resultados anteriores no permiten atribuir a un canal de transmisión específico las posibles fuentes del efecto industrial diferenciado ante variaciones permanentes y sorpresivas en la política monetaria del Banco de México. En el caso del modelo 1, conjuntar las actividades dedicadas a la producción de bienes duraderos y no duraderos parece explicar la falta de significación estadística de las variables que representan ambos canales de transmisión. Por otro lado, cuando se distinguen las actividades que producen bienes no duraderos en los modelos 2 y 3 , los resultados sugieren que los canales de la tasa de interés y del crédito parecen no funcionar significativamente a través de este tipo de actividades; este resultado concuerda con los hallazgos en la literatura internacional mencionada.

Con el propósito de analizar si las actividades manufactureras que producen bienes duraderos pudieran representar un vehículo para explicar las respuestas heterogéneas a través de ambos canales de transmisión de la política monetaria, se modificaron las especificaciones en los modelos 4 y 5 para incluir las medidas de intensidad de capital y de tamaño de los establecimientos para las actividades que producen este tipo de bienes. Los resultados de los estadísticos de contraste sugieren que las especificaciones en ambos modelos son razonables. En particular, cuando se compara el modelo 4 con el modelo 5, que incluye una medida para las empresas grandes, puede considerarse que este último 
anida al primero y los estadísticos de log-verosimilitud (LV) y HannanQuinn favorecen al modelo 5; algo similar ocurre cuando se comparan los modelos 5 y 1 . Conforme a las estimaciones en el modelo 5, las variables globales que miden la intensidad en el uso de capital (I/VA) y la proporción de micro y pequeños establecimientos son estadísticamente significativos a $10 \%$. El signo positivo en ambos casos sugiere que las actividades manufactureras con una relativa mayor intensidad en el uso de capital son aquéllas que en promedio reciben el mayor efecto de las variaciones en la política monetaria mediante el canal de la tasa de interés. Por su parte, las actividades manufactureras con una proporción mayor de establecimientos micro y pequeños parecen recibir los mayores efectos de la política monetaria a través del canal del crédito.

Por otro lado, la variable que mide la intensidad en el uso de capital únicamente en las actividades que producen bienes duraderos tiene una asociación significativa aún mayor que para el conjunto del sector manufacturero. Este resultado confirma la importancia de las actividades que producen bienes duraderos para explicar cuáles son las vías por las que la política monetaria puede tener mayor incidencia. En este caso, una variación en la política monetaria tendría un efecto hasta tres veces mayor en la producción que realizan este tipo de actividades, sobre el conjunto del sector manufacturero, con un coeficiente para los subsectores que producen bienes durables de $0.038 .{ }^{4}$ Con relación a la variable que mide la proporción de establecimientos de tamaño micro y pequeños en la producción de bienes duraderos, el coeficiente estimado resultó significativo a $1 \%$, aunque con signo negativo, lo que indica que el efecto de la política monetaria sobre la producción industrial a través del canal de crédito ocurriría en los subsectores de bienes duraderos, aunque aparentemente en menor medida en aquéllos cuya presencia de micro y pequeños establecimientos es relativamente menor. Este último rasgo puede estar relacionado con el limitado acceso a fuentes de financiamiento formales que el sector de micro y pequeñas empresas han enfrentado en el país (Dussel, 2004), particularmente en el segmento de microempresas, situación que las haría menos sensibles a variaciones sorpresivas en la tasa de interés. Por otro lado, el contar con

4 El coeficiente resulta de sumar el coeficiente estimado para la variable interactiva I/VA durables (0.028) con el coeficiente de la variable global I/VA (0.0102). 
alternativas de financiamiento que puedan complementar el financiamiento formal, tales como el proveniente de proveedores, de familiares y amigos, de programas públicos, entre otros, puede servir como un factor estabilizador en los casos cuando un aumento sorpresivo en el costo del financiamiento formal pueda poner en riesgo la operación del establecimiento. $^{5}$

\section{CUADRO 4}

FUENTES DEL EFECTO INDUSTRIAL HETEROGÉNEO DE LA POLÍTICA MONETARIA EN MÉXICO

\begin{tabular}{|c|c|c|c|c|c|}
\hline \multicolumn{6}{|c|}{ Variable dependiente: Impacto acumulado medio (valores absolutos) } \\
\hline Variables & Modelo 1 & Modelo 2 & Modelo 3 & Modelo 4 & Modelo 5 \\
\hline Constante & 0.178 & 0.169 & 0.036 & -0.045 & -0.134 \\
\hline Estadístico $t$ & $1.974^{*}$ & $2.657^{* *}$ & 0.156 & 0.493 & -0.645 \\
\hline I/VA & 0.003 & -0.006 & -0.0002 & 0.01 & 0.0102 \\
\hline Estadístico $t$ & 0.369 & -0.687 & -0.028 & 1.652 & $1.779 *$ \\
\hline EMP & -0.0009 & 0.006 & 0.0045 & 0.004 & 0.0047 \\
\hline Estadístico t & -0.445 & $1.847^{*}$ & 0.999 & $2.51 * *$ & $1.836^{*}$ \\
\hline I/VA durables & & & & 0.033 & 0.028 \\
\hline Estadístico $t$ & & & & $6.369 * * *$ & $6.851 * * *$ \\
\hline EMP durables & & & & -0.009 & -0.0081 \\
\hline Estadístico $t$ & & & & $-4.339 * * *$ & $-4.200 * * *$ \\
\hline I/VA no durables & & 0.007 & 0.011 & & \\
\hline Estadístico t & & 1.113 & 1.121 & & \\
\hline EMP no durables & & -0.007 & -0.005 & & \\
\hline Estadístico t & & $.0648^{*}$ & -1.045 & & \\
\hline EG & & & 0.001 & & 0.0015 \\
\hline Estadístico t & & & 0.453 & & 0.6708 \\
\hline R-cuadrada & 0.023 & 0.222 & 0.212 & 0.744 & 0.877 \\
\hline R-cuadrada ajustada & -0.086 & 0.014 & -0.07 & 0.676 & 0.833 \\
\hline Estadístico F & 0.209 & 1.069 & 0.751 & 10.905 & 19.985 \\
\hline Prob. Estadístico F & 0.813 & 0.406 & 0.599 & 0.0002 & $6.35 \mathrm{E}-06$ \\
\hline Estadístico LV & -43.414 & -30.680 & -34.418 & -39.415 & -33.793 \\
\hline Hannan-Quinn & 93.507 & 72.332 & 82.002 & 89.802 & 80.752 \\
\hline
\end{tabular}

Fuente: elaborado con estimaciones propias.

5 Información obtenida a partir de la Encuesta Nacional de Financiamiento de las Empresas (2018) muestra que, del total de micro y pequeñas empresas encuestadas, el 75\% y $70 \%$ declararon respectivamente haber tenido un crédito con una institución bancaria. Sin embargo, el $45 \%$ y $52 \%$ en este mismo grupo de empresas encuestadas, también indicaron haber accedido a financiamiento mediante vías no formales (familiares y amigos, proveedores) e inclusive a través de programas públicos. 


\section{CONCLUSIONES}

En este documento se ha analizado el efecto que un cambio permanente de tipo contractivo en la política monetaria tendría en el nivel de producción y de precios en cada uno de los subsectores que conforman la actividad manufacturera en el país. Los resultados señalan importantes efectos diferenciados en el impacto de este tipo de políticas a nivel desagregado, tanto en magnitud como en el horizonte temporal. Esto implica, por un lado, la política monetaria es no neutral en su efecto sobre la producción industrial y, por otro, que ésta puede ser efectiva para la estabilización del nivel de precios en el corto y largo plazos.

En este sentido, ante un choque positivo sobre la tasa de interés, los subsectores ubicados en el primero y segundo cuartil muestran un comportamiento similar, con un ajuste gradual de sus precios y con un ligero aumento al inicio, alcanzando su menor valor dos años y medio después del momento de inicio del choque. Por su parte, las industrias ubicadas en el tercer cuartil resultan menos sensibles a este tipo de efectos. En el caso de la producción, la magnitud de los ajustes es más significativa en comparación con la de los precios. La contracción de la producción de las industrias ubicadas en los dos primeros cuartiles es de magnitud pequeña al inicio del choque, y alcanza su mayor caída 36 meses después. Por el contrario, la evidencia muestra que las industrias ubicadas en el tercer cuartil son menos sensibles a los movimientos de la tasa de interés.

Adicionalmente, el análisis acerca de las posibles fuentes de los efectos industriales observados sugiere que el canal de la tasa de interés y del crédito serían relevantes para explicar por qué algunos sectores son más afectados que otros ante las variaciones inesperadas de la política monetaria. Los resultados indican que es a través de los subsectores que producen bienes duraderos, la vía por la cual el Banco de México puede tener mayor influencia en la actividad económica real en el corto plazo a través del canal tradicional de la tasa de interés, así como también demuestra la evidencia cuando se considera la medida de intensidad en el uso de capital. No obstante, el rol que parecen tener las asimetrías en la información disponible entre prestamistas y prestatarios también parece otorgar relevancia al canal de crédito. Aunque el tamaño de las empresas sugiere que las limitaciones para acceder a créditos ampli- 
ficarían el impacto de la política monetaria en sectores con mayor proporción de empresas micro y pequeñas, en algunos casos, el acceso a alternativas de financiamiento consideradas no formales puede servir para estabilizar o amortiguar el impacto en las actividades dedicadas principalmente a la producción de bienes duraderos.

De esta forma, este documento aporta evidencia acerca de los efectos diferenciados de los cambios en el instrumento de la política monetaria en los subsectores manufactureros y sus posibles canales de transmisión a partir de características específicas de la estructura industrial. En conjunto, esta evidencia sugiere que se vuelve relevante el trazar los efectos de la política monetaria no solamente para las variables económicas agregadas, sino también a un nivel de desagregación sectorial, con la finalidad de proveer información que facilite la coordinación con otras medidas de política económica, cuando la política monetaria no tenga la efectividad esperada. Adicionalmente, los resultados sugieren la posibilidad de transitar hacia un esquema de política monetaria que no solamente considere la estabilidad de precios como objetivo principal, sino también como promotor del impulso a la actividad económica real.

\section{BiBLIOGRAFÍA}

Alam, Tasneem y Muhammad Waheed (2006). "Sectoral effects of monetary policy: evidence from Pakistan", The Pakistan Development Review, 45(4), pp. 1103-1115.

Bernake, Ben y Mark Gertler (1995). "Inside the black box: The credit channel of monetary policy", NBER Working Paper No. 5146, National Bureau of Economic Research.

Bravo, Héctor;Carlos García, Verónica Mies y Matías Tapia (2003). "Heterogeneidad de la transmisión monetaria: efectos sectoriales y regionales", Economía Chilena, 6(3), pp. 5-26.

Carbajal Suárez, Yolanda y Berenice Carrillo Macario (2017). "Producción y empleo manufacturero en las entidades federativas de México, 19882013", en Yolanda Carbajal Suárez y Leobardo de Jesús Almonte (coords.). Dinámica del empleo y la producción manufacturera en México, UAEM / Ediciones y Gráficos Eón, México, pp. 15-42.

Carlino, Gerald y Robert H. DeFina (1995). "The differential effects of monetary policy shocks on regional economic activity," Working Papers 95-15, 
Federal Reserve Bank of Philadelphia.

Dedola, Luca y Franceso Lippi (2005). "The monetary transmission mechanism: evidence from the industries of five OECD countries", European Economic Review 49, pp. 1543-1569.

De Jesús Almonte, Leobardo (2019). Lento crecimiento y empleo manufacturero en México. Un análisis de endogeneidad territorial, UAEM / Ediciones y Gráficos Eón, México.

Dussel, Enrique (2004). "Pequeña y mediana empresa en México: condiciones, relevancia en la economía y retos de política", Economía UNAM, 1(2), pp. 64-84.

Garcia-Herrero, Alicia, Eric Girardin y Arnoldo López-Marmolejo (2019), "Mexico's monetary policy communication and money markets", International Journal of Economics and Finance 11(2), pp. 81-97.

Ganley, Joe y Chris Salmon (1997). "The industrial impact of monetary policy shocks: some stylised facts", Working Paper 68, Bank of England.

Gertler, Mark y Simon Gilchrist (1994). "Monetary policy, business cycles, and the behavior of small manufacturing firms", The Quarterly Journal of Economics, 109(2), pp. 309-340.

Georgopoulos, George y Walid Hejazi (2009). "Financial structure and heterogenous impact of monetary policy across industries", Journal of Economics and Business, 61, pp. 1-33.

Hayo, Bernd y Birgit Uhlenbrock (1999). "Industry effects of monetary policy in Germany", ZEI Working Paper No. B 14-1999, Rheinische FriedrichWilhelms-Universität Bonn, Zentrum für Europäische Integrationsforschung (ZEI), Bonn.

Ibarra, Raul (2016). "How important is the credit channel in the transmission of monetary policy in Mexico?", Applied Economics, 48(36), pp. 34623484.

Ibrahim, Mansor y Ruzita Mohd (2005). "Exchange rate, monetary policy and manufacturing output in Malaysia", Journal of Economic Cooperation, 26 (3), pp. 103-130.

Im, Kyung, M. Hashem Pesaran y Yongcheol Shin (2003). "Testing for unit roots in heterogeneous panels", Journal of Econometrics, 115(1), pp. 5374.

Kutu, Adebayo y Harold Ngalawa (2016). "Monetary policy shocks and industrial sector performance in South Africa", Journal of Economics and Behavorial Studies, 8(3), pp. 26-40.

Lee, Junsoo y Mark Strazicich (2013). "Minimum LM unit root test with one structural break", Economics Bulletin, 33(4), pp. 2483-2492.

Rodríguez Medina, Oscar y Elías Alvarado Lagunas (2016). Inflation targeting and policy rules: The case of Mexico, 2001-2012, Apple Academic Press y CRC Press, Estados Unidos.

OECD (2019). Estudios Económicos de la OECD: México 2019, París. 
OECD (2017). Estudios Económicos de la OECD: México 2017, París.

Pedroni, Peter (2013). "Structural Panel VARs", Econometrics, 1(2), pp. 1-27.

Peersman, Gert y Frank Smets (2005). "The industry effects of monetary policy in the Euro Area", The Economic Journal, 115, pp. 319-342.

Quintero, Jorge (2017). "Industrial structure and transmission of monetary policy in Latin American Countries", Investigación Económica, 34(302), pp. 103-129.

Raddatz, Claudio y Robert Rigobon (2003). "Monetary policy and sectoral shocks: did the Fed react properly to the high-tech crisis?", Working Paper 9835, National Bureau of Economic Research, Cambridge, MA.

Rodríguez-Fuentes, Carlos y David Padrón-Marrero (2008). "Industry effects of monetary policy in Spain", Regional Studies, (42) 3, pp. 375-384.

Sengupta, Nandini (2014). "Sectoral effects of monetary policy in India", South Asian Journal of Macroeconomics and Public Finance, 3(1), pp. 127-154.

Torres-Preciado, Víctor Hugo (2017). "Política monetaria, producción y empleo manufacturero en México", Revista de Economía, 34(88), pp. 9-44.

Vespignani, Joaquín L. (2012). "The industrial impact of monetary shocks during the inflation targeting era in Australia", School of Economics and Finance, Discussion Paper 12, University of Tasmania. 